\title{
Cause of mortality in aortoesophageal fistula: oesophageal sepsis. A case report
}

\author{
Ulaş Aday, Durmuş Ali Çetin, Hüseyin Çiyiltepe, Ebubekir Gündeş, Emre Bozdağ, Aziz Serkan Senger \\ Department of Gastroenterological Surgery, Kartal Kosuyolu High Speciality Education and Training Hospital, Istanbul, Turkey
}

Gastroenterology Rev 2017; 12 (3): 222-225

DOI: https://doi.org/10.5114/pg.2017.70476

Address for correspondence: Ulaş Aday, Department of Gastroenterological Surgery, Kartal Kosuyolu High Speciality Education and Training Hospital, 34685 Istanbul, Turkey, phone: +90 5302933895, e-mail: ulasaday@gmail.com

Aortoesophageal fistula (AEF) is a rare condition, which causes massive upper gastrointestinal system bleeding [1]. The most frequent cause of primary AEF is thoracic aortic aneurysm, which is engendered by such causes as the rupture of penetrating aortic ulcer, foreign body swallowing, thoracic trauma, oesophageal cancer, and bronchogenic carcinoma. Secondary AEF, on the other hand, is frequently caused by surgical procedures done by graft because of aortic aneurysms or neighbouring organ surgeries [2, 3]. Emergency surgical procedures to treat life-threatening massive bleeding following AEF have a high risk of mortality. Therefore, thoracic endovascular aortic repair (TEVAR) has recently become a popular and effective method [4]. It has been reported that the most significant clinical condition affecting patients' long-term survival following the achievement of bleeding control through TEVAR was continuing oesophageal fistula and the related sepsis secondary to mediastinitis. Thus, it has been suggested that definitive surgical procedures to treat the oesophagus should be performed without delay following the stabilisation of the patient [5]. The surgical procedure to be performed varies according to the underlying causes. The control of the continuing infection and the related sepsis has been considered to be the Achilles' heel of treatment [6].

This study presents the case of a patient with AEF, whose bleeding control was achieved through TEVAR, but who died of oesophageal sepsis. The goal of the study is to underline the significance of the oesophagus in clinical profile and the control of the source of infection in order to decrease the rate of mortality.

A 59-year-old female patient had been evaluated at a different centre because of haematemesis, which had started three days earlier, and had been referred to our hospital's cardiovascular surgery clinic diagnosed with AEF. The patient, who had a history of diabe- tes for about 25 years, had no history of surgery. The first clinical evaluation of the patient revealed arterial blood pressure level of 108/68 $\mathrm{mm} \mathrm{Hg}$, heart rate of $112 \mathrm{~min}^{-1}$, and a normal neurological examination result. Her rectal evaluation showed melena. The patient was taken into intensive care and was monitored. Her laboratory results were as follows: hematocrit $22.4 \%$ (normal range: 33-54\%), hemoglobin $7.8 \mathrm{~g} / \mathrm{dl}$ (normal range: $11.1-17.1 \mathrm{~g} / \mathrm{dl}$ ), urea $100 \mathrm{mg} / \mathrm{dl}$ (normal range: 10-50 mg/dl), albumin $1.87 \mathrm{~g} / \mathrm{dl}$ (normal range: 3.5$5.2 \mathrm{~g} / \mathrm{dl}$ ), blood glucose $363 \mathrm{mg} / \mathrm{dl}$ (normal range: 70$105 \mathrm{mg} / \mathrm{dl}$ ), and C-reactive protein (CRP) $12.5 \mathrm{mg} / \mathrm{dl}$ (normal range: $0-0.4 \mathrm{mg} / \mathrm{dl}$ ). Other laboratory results were within normal limits. Following blood replacement (a total of 9 units of erythrocyte suspension, with 5 before arriving at our centre and 4 at our intensive care unit) and hydration, TEVAR was planned and oral + intravenous contrasted computed tomography (CT) was performed. Computed tomography results showed an AEF line developed at the base of the saccular aneurysm in the thoracic aorta and extravasation of the oral contrasted fluid administered before the intravenous contrast. A defect and dilatation were seen on the oesophageal wall (Figures 1 A, B). Bleeding was brought under control through TEVAR and total parenteral nutrition, low-molecular-weight heparin prophylaxis, and antifungal agent alongside broad-spectrum antibiotherapy were initiated. Although there was a current in the left subclavian artery following the completion of the TEVAR procedure (Figure 2), temporary loss of strength was seen in the upper and lower left extremities a day after the procedure. The patient's neurological evaluation revealed neither intracranial bleeding nor signs pertaining to infarction. Her neurological symptoms recovered in the following days.

Endoscopic examination for the oesophageal gap was performed for the patient, whose clinical condition 

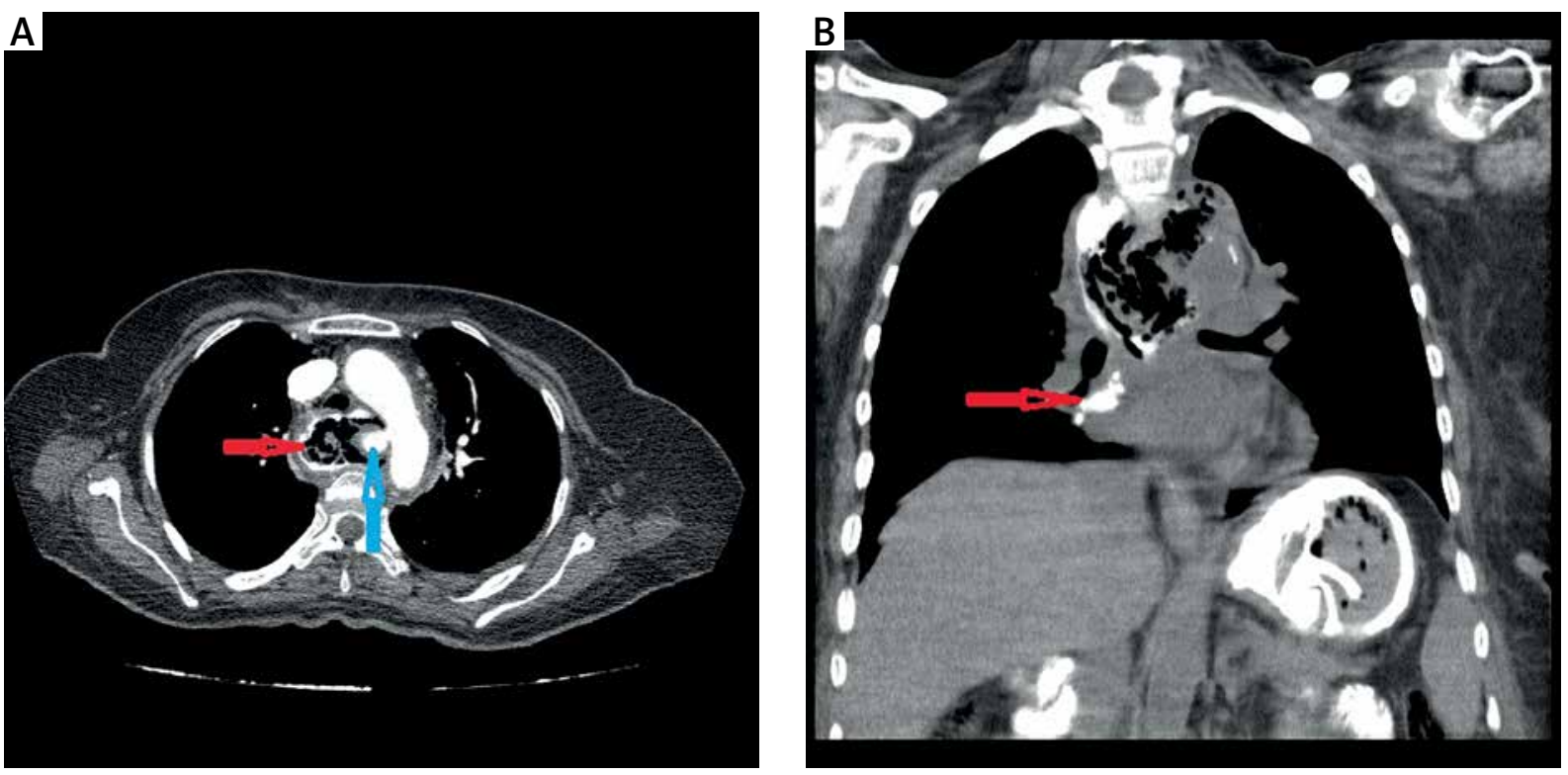

Figure 1. A - Image of computed tomography. Defect and dilatation were seen on the oesophageal wall (red arrow), saccular aneurysm in the thoracic aorta (blue arrow). B - Image of computed tomography. Extravasation of the oral contrasted fluid in mediastinum

was stable, and infective parameters regressed. Her endoscopic results obtained on the $17^{\text {th }}$ day of the follow-up demonstrated a wall defect of about $23-27 \mathrm{~cm}$ on the oesophagus (Figure $3 \mathrm{~A}$ ). There was infected material between the aortic graft and the oesophagus. An endoscopic self-expendable metallic stent was placed in order to prevent continuing contamination (Figure 3 B). Definitive surgery was planned for the patient, but she died on the $28^{\text {th }}$ day of follow-up because of multi-organ failure (MOF) related to progressive sepsis.

The AEF is one of the rare causes of upper digestive system bleedings. It is frequently caused by thoracic aortic aneurysm (54\%), foreign body swallowing (19\%), or oesophageal or bronchial carcinoma (17\%), and previous history of surgical procedures [7]. It generally cannot be diagnosed before the presence of massive bleeding. Endoscopy and contrasted tomography performed in order to explore the cause of bleeding enable diagnosis. An impression of a mass at mid-oesophagus can be seen during endoscopy but no biopsy samples should be taken, in order to avoid massive bleeding [1]. The first measure that should be taken in treatment is to haemodynamic stabilisation of the patient through bleeding control. The mortality rates of emergency surgical procedures are quite high (45-55\%). Therefore, TEVAR has recently become a frequently performed method because it is minimally invasive and enables rapid and effective control of bleeding [4]. However, the graft, which is placed during endovascular repair, gives way to an increase in ischaemia by decreasing the blood

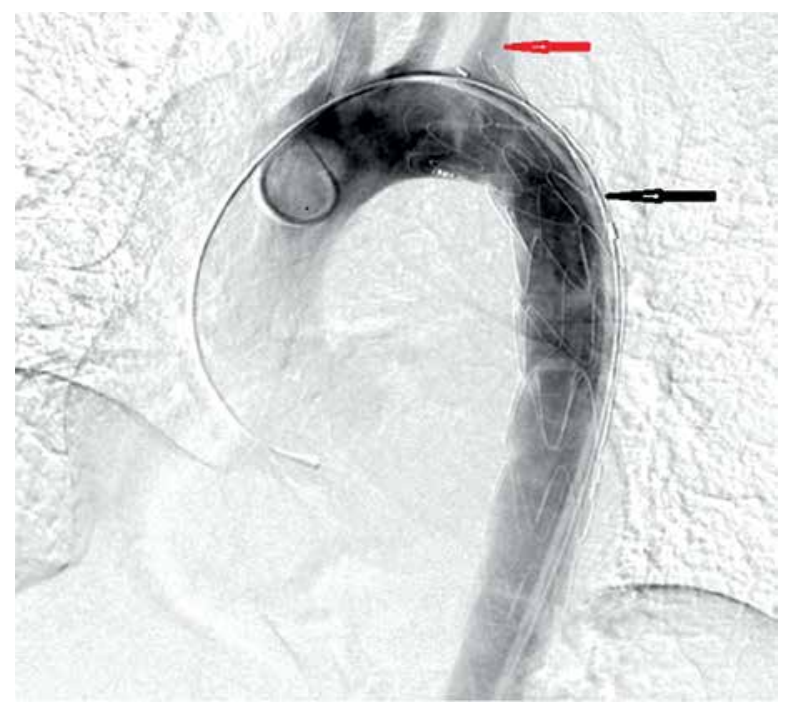

Figure 2. Image of angiography, completion of the TEWAR procedure.

Black arrow - stent, red arrow - left subclavian artery.

circulation of the oesophagus, it causes enlargement of the gap by mechanical pressure, and the up and down movement of the graft through the contraction of the heart deteriorates the traumatic effect on the oesophageal wall. The combination of the infected area and these effects contributes to the enlargement of the defect on the oesophagus [8]. In spite of the fact that TEVAR effectively controls bleeding, it also negatively 

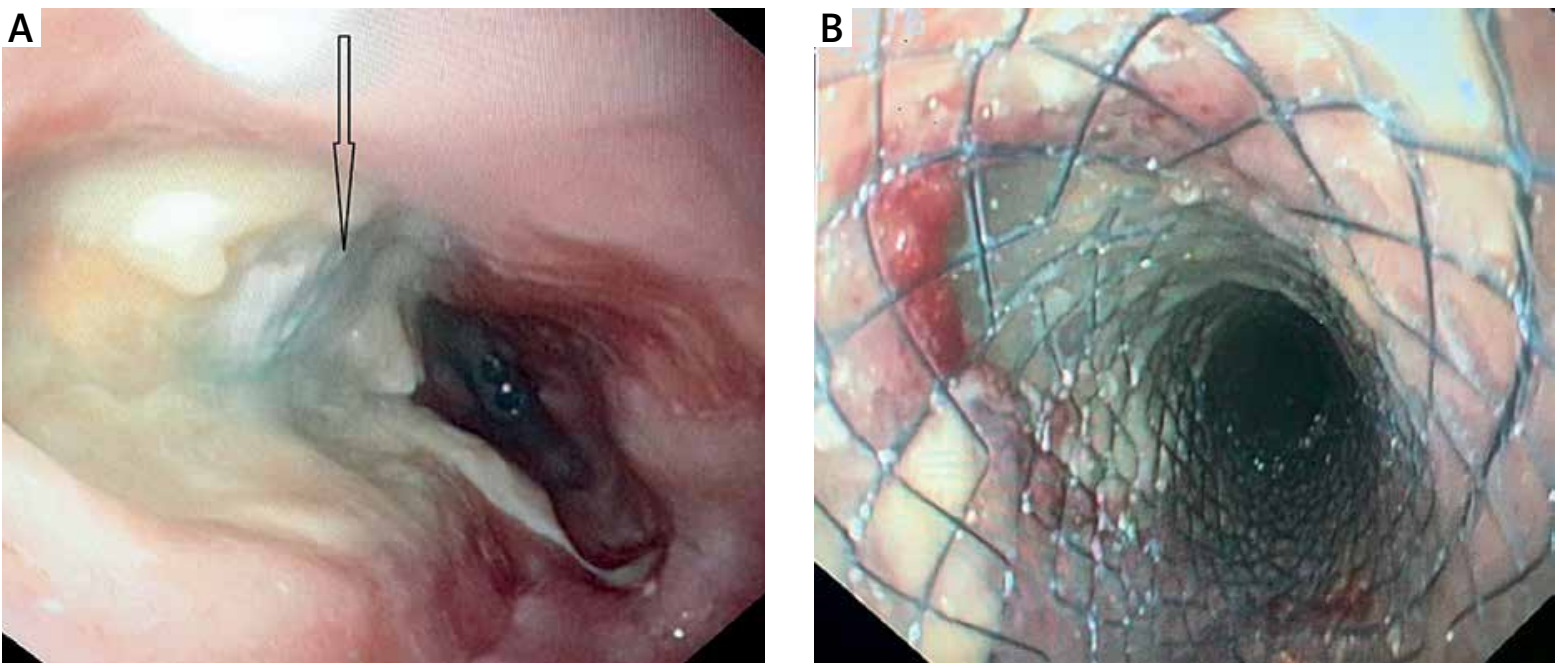

Figure 3. A - Endoscopic examination of the oesophagus. Wall defect of about $23-27 \mathrm{~cm}$ on the oesophagus aortic graft was seen (arrow). B - Image of endoscopic self-expendable metallic stent in oesophagus

affects the oesophageal defect; therefore, treatment of the oesophagus should be planned without any delay following the achievement of haemodynamic stability. In a review by Canaud et al. [4], the authors reported $52.5 \%$ mortality rate in cases with AEF with sole use of TEVAR. Thus, many studies have recommended that TEVAR should be utilised as a bridge treatment to stabilise the patient prior to definitive treatment [2, 4-6].

Endoscopic stent practice is frequently used for the malign obstructions of the oesophagus for palliative purposes. But the frequency of its utilisation for the treatment of leaks that take place after upper digestive system surgeries has risen in recent years [9]. It has such advantages as being minimally invasive because of its endoscopic applicability, a high rate of success, and being able to be removed. It can enable the control of the source of infection by preventing the access of the oesophageal content into the extraluminal area, as well as enabling healing in cases with smaller wall defects. As the stent's migration downwards from the area of the defect is relatively frequent, it has been suggested that its localisation should be checked at intervals $[10,11]$. In our case, the patient's endoscopic evaluation revealed that the oesophageal defect was rather wide, and it was observed that the graft, which moved with the pulse from the area of the defect, had a traumatic effect on the wall. There were also infected fluid and ischaemic tissue elements between the graft and the oesophageal gap. Although a self-expandable metallic stent was placed into the oesophagus endoscopically, the patient's deterioration to multi-organ failure related to sepsis could not be prevented. The fact that the patient's clinical condition was stable and the regression in her infection parameters within the 2 -week period following the control of bleeding was misleading and led to a delay in the intervention into the oesophagus. In AEF treatment, the prevention of contamination by placing a stent into the oesophagus during the early period following the control of bleeding can be utilised as a bridging treatment for the oesophagus until the performance of definitive surgery. Especially in cases where the general condition of the patient is not eligible for surgical procedures, the placement of a stent into the oesophageal defect during the early period may prove to be a rational approach.

In $A E F$, the surgical procedure that can be planned for the oesophagus varies depending on the underlying pathology, the condition of the patient, and the experience of the centre. Topel et al. [2] repeated thoracotomy covering oesophageal resection because of continuing leaks in 3 out of 4 cases with AEF, for whom they had performed primary oesophageal repair through omental flaps. Akashi et al. [5] reported in their study conducted with 47 cases that the best long-term survival was achieved in patients who had undergone esophagostomy alongside replacement of the infected aorta with a graft. In this study, only 5 (20.8\%) out of 24 patients who had been diagnosed with primary AEF reached 18-month survival. Successful results have also been reported in cases where the oesophageal defect was supported following repair or through intercostal or pectoral muscular flaps in oesophageal reconstruction [3, 12]. The majority of the mortality cases are related to infection. Therefore, immediate initiation of broad-spectrum antibiotherapy and the performance of definitive surgery in patients eligible for surgical procedures following the control of bleeding as soon as possible will significantly decrease the rate of mortality [4, 5, 13]. 
Multistage surgery with subtotal oesophagostomy, proximal oesophagostomy, and feeding jejunostomy followed by a reconstruction with the stomach, colon, jejunum, etc. is a frequently utilised method as well [14].

The AEF is a rare but life-threatening condition through massive aortic bleeding and oesophageal infection. The TEVAR is a minimally invasive method that enables the rapid, effective, and safe control of bleeding, but it may have negative effects on oesophageal recovery. The control of oesophageal infection is highly significant in long-term survival. Stent placement into the oesophagus during the early phase can contribute to the control of the source of infection. Surgical intervention still proves to be the most outstanding mode of treatment and it should be performed in stable patients as soon as possible.

\section{Conflict of interest}

The authors declare no conflict of interest.

\section{References}

1. Reardon MJ, Brewer RJ, LeMaire SA, et al. Surgical management of primary aortoesphageal fistula secondary to thoracic aneurysm. Ann Thorac Surg 2000; 69: 967-70.

2. Topel I, Stehr A, Steinbauer MG, et al. Surgical strategy in aortoesophageal fistulae: endovascular stent grafts and in situ repair of the aorta with cryopreserved homografts. Ann Surg 2007; 246: 853-9.

3. Arab WA, Chagnon F, Echave V, Sirois M. A multidisciplinary approach to aortoesophageal fistula: a case report. Turkish J Thorac Cardiovasc Surg 2011; 19: 285-87.

4. Canaud L, Ozdemir BA, Bee WW, et al. Thoracic endovascular aortic repair in management of aortoesophageal fistulas. J Vasc Surg 2014; 59: 248-54.

5. Akashi H, Kawamoto S, Saiki Y, et al. Therapeutic strategy for treating aortoesophageal fistulas. Gen Thorac Cardiovasc Surg 2014; 62: 573-80.

6. Mosquera VX, Marini M, Pombo-Felipe F, et al. Predictors of outcome and different management of aortobronchial and aortoesophageal fistulas. J Thorac Cardiovasc Surg 2014; 148: 3020-6.

7. Barrios Carvajal M, Díaz-Tobarra M, Martí-Obiol R, et al. Combined treatment of an aortoesophageal fistula after aortoplasty for aortic stenosis. Ann Thorac Surg 2015; 100: 1091-3.

8. Dumfarth J, Dejaco H, Krapf C, et al. Aorto-esophageal fistula after thoracic endovascular aortic repair: successful open treatment. Aorta (Stamford) 2014; 2: 37-40.

9. Leenders BJ, Stronkhorst A, Smulders FJ, et al. Removable and reposition able covered metal self-expandable stents for leaks after upper gastrointestinal surgery: experiences in a tertiary referral hospital. Surg Endosc 2013; 27: 2751-9.

10. vanHeel NC, Haringsma J, Wijnhoven BP, Kuipers EJ. Endoscopic removal of self-expandable metal stents from the esophagus (with video). Gastrointest Endosc 2011; 74: 44-50.
11. Schweigert M, Dubecz A, Stadlhuber RJ, et al. Treatment of intrathoracic esophageal anastomotic leaks by means of endoscopic stent implantation. Interact Cardiovasc Thorac Surg 2011; 12: 147-51.

12. Marone EM, Coppi G, Kahlberg A, et al. Combined endovascular and surgical treatment of primary aortoesophageal fistula. Tex Heart Inst J 2010; 37: 722-4.

13. Malas MB, Saha S, Qazi U, et al. Is endovascular stent-graft treatment of primary aortoesophageal fistula worthwhile? Vasc Endovascular Surg 2011; 45: 83-9.

14. Göbölös L, Miskolczi S, Pousios D, et al. Management options for aorto-oesophageal fistula: case histories and review of the literature. Perfusion 2013; 28: 286-90.

Received: 18.04 .2017

Accepted: 25.04.2017 